\title{
Bifurcation analysis of a modified cytotoxic T-lymphocyte response model with two delays
}

\author{
Qingshui Liao ${ }^{\text {a,** }}$ \\ Guizhou Normal University, Guiyang, Guizhou, China \\ a'Email:qsliao_math@163.com \\ *Qingshui Liao
}

Keywords: cytotoxic T-lymphocyte, stability, periodic solution, delay, Hopf bifurcation.

\begin{abstract}
In this paper, a modified cytotoxic T-lymphocyte response model with two delays is considered. By regarding the delays as the bifurcation parameter, the local asymptotic stability of the positive equilibrium is studied. We found that the system undergoes a Hopf bifurcation of a nonconstant periodic solution at positive equilibrium when the delays across through a sequence of bifurcated periodic solutions. And, we investigate the direction and the stability of bifurcated periodic solution.
\end{abstract}

\section{Introduction}

Recently, population models of immune systems have been studied extensively. The cytotoxic Tlymphocyte has plays an important role in medicine science, and it has been widely studied by many researchers (see, e.g. [1,2] and the references therein). In $[3,4]$ the following differential equations system was considered

$$
\left\{\begin{array}{l}
x_{1}^{\prime}=\gamma-d x_{1}-\beta x_{1} x_{2}, \\
x_{2}^{\prime}=\beta x_{1} x_{2}-a x_{2}-p x_{2} x_{3}, \\
x_{3}^{\prime}=c x_{2} x_{3}-b x_{3} .
\end{array}\right.
$$

It is of necessary to incorporate time delays into the models for reflecting the dynamical behaviours. So in this paper, we investigate the CTL response system with two delays, as follows

$$
\left\{\begin{array}{l}
x_{1}^{\prime}=\gamma-d x_{1}-\beta x_{1} x_{2}\left(t-\tau_{1}\right), \\
x_{2}^{\prime}=\beta x_{1}\left(t-\tau_{2}\right) x_{2}-a x_{2}-p x_{2} x_{3}, \\
x_{3}^{\prime}=c x_{2} x_{3}-b x_{3} .
\end{array}\right.
$$

where $\gamma, \beta, d, a, p, c, b$ are positive constants, $x_{1}, x_{2}, x_{3}, \tau_{1}, \tau_{2}$, are functions respect to $t$. It is well known that periodic solutions can arise through the Hopf bifurcation [5-7] in delay differential equations. Therefore, in this paper, we take bifurcation phenomenon and the properties of periodic solutions of system (1.2) into consideration.

The organization of this paper is as follows. In section 2, we discuss the stability of the positive equilibrium and the existence of Hopf bifurcation. In section 3, the direction of Hopf furcation and the stability of bifurcated periodic solutions are determined.

\section{Stability of positive equilibrium and Hopf bifurcation}

It is easy to see that system (1.2) has three equilibrium. Following are the equilibrium states:

$$
\begin{aligned}
& A: E_{1}\left(m_{1}, 0,0\right), m_{1}=\frac{\gamma}{d} ; \quad B: E_{2}\left(m_{2}, m_{3}, 0\right), \quad m_{2}=\frac{a}{\beta} ; m_{3}=\frac{(\gamma \beta-a d)}{a \beta} ; \\
& C: E^{*}\left(x_{1}^{*}, x_{2}^{*}, x_{3}^{*}\right), \quad x_{1}^{*}=\frac{c \gamma}{c d+b \beta}, \quad x_{2}^{*}=\frac{b}{c}, \quad x_{3}^{*}=\frac{[c \gamma \beta-a(c d+b \beta)]}{p(c d+b \beta)} .
\end{aligned}
$$

For convenient, we take the following notes.

$$
\left(H_{1}\right): c \gamma \beta>a(c d+b \beta),\left(H_{2}\right):\left(d+\beta x_{2}^{*}\right)^{2}>2 c p x_{2}^{*} x_{3}^{*},\left(H_{3}\right):\left(c p x_{2}^{*} x_{3}^{*}\right)^{2}>2 c p\left(d+\beta x_{2^{*}}\right) x_{2}^{*} x_{3}^{*}+\left(\beta^{2} x_{1}^{*} x_{2}^{*}\right)^{2} .
$$

If system (1.2) satisfies $\left(H_{1}\right)$, it has a unique positive equilibrium and two boundary equilibria. Now, we do investigate the stability of the positive equilibrium. 
Let $u_{1}(t)=x_{1}\left(t-\tau_{2}\right), u_{2}(t)=x_{2}(t), u_{3}(t)=x_{3}(t), \tau=\tau_{1}+\tau_{2}$ then system (1.2) becomes

$$
\left\{\begin{array}{l}
u_{2}^{\prime}(t)=\gamma-d u_{1}(t)-\beta u_{1}(t) u_{2}(t-\tau), \\
u_{1}^{\prime}(t)=\beta u_{1}(t) u_{2}(t)-a u_{2}(t)-p u_{2}(t) u_{3}(t), \\
u_{3}^{\prime}(t)=c u_{2}(t) u_{3}(t)-b u_{3}(t) .
\end{array}\right.
$$

The characteristic of $(2.1)$ at $E^{*}$ is

$$
\lambda^{3}+\lambda^{2}\left(d+\beta x_{2}^{*}\right)+\lambda c p x_{2}^{*} x_{3}^{*}+\lambda \beta^{2} x_{1}^{*} x_{2}^{*} e^{-\lambda \tau}+c p\left(d+\beta x_{2}^{*}\right) x_{2}^{*} x_{3}^{*}=0 .
$$

Let $M_{1}=d+\beta x_{2}^{*}, M_{2}=c p x_{2}^{*} x_{3}^{*}, M_{3}=\beta^{2} x_{1}^{*} x_{2}^{*}$. Then, we arrive at

$$
\lambda^{3}+M_{1} \lambda^{2}+M_{2} \lambda+M_{3} e^{-\lambda \tau} \lambda+M_{1} M_{2}=0 .
$$

In the following, we regard the time delay as the parameter to consider the local stability of the positive equilibrium and the Hopf bifurcation of the system (2.1).

Lemma 2.1 Assume that $\left(H_{1}\right)$ is hold. Then, the positive equilibrium $E^{*}\left(x_{1}^{*}, x_{2}^{*}, x_{3}^{*}\right)$ of system is locally asymptotically stable in the absence of delay.

Proof When $\tau=0,(2.3)$ reduces to

$$
\lambda^{3}+M_{1} \lambda^{2}+\left(M_{2}+M_{3}\right) \lambda+M_{1} M_{2}=0 .
$$

Clearly, by Routh-Hurwitz criterion, we know that all the roots of (2.4) have negative real parts. Then, the conclusion is obtained.

Lemma 2.2 Suppose that $\left(H_{1}\right),\left(H_{2}\right)$ and $\left(H_{3}\right)$ is satisfied. Then, at

$$
\tau_{j}=\frac{1}{\omega_{0}} \arccos \left[\frac{M_{1} \omega_{0}^{2}-M_{1} M_{2}}{\omega_{0}^{3}-M_{2} \omega_{0}}\right]+\frac{j \pi}{\omega_{0}}, j=0,1,2 \ldots
$$

(2.3) has a simple pair of conjugate purely imaginary roots $\pm i \omega_{0}$, where $\omega_{0}=d$.

Furthermore, we have the following results

(i) If $\tau \in\left[0, \tau_{0}\right)$, then all the roots of (2.3) have negative real parts;

(ii) If $\tau=\tau_{0},\left(H_{2}\right)$ and $\left(H_{3}\right)$ is satisfied then (2.3) has a simple pair of conjugate purely imaginary roots $\pm i \omega_{0}$, and all other roots have negative real parts.

Proof Now for $\tau>0$, let $\lambda=i \omega$ is a root of (2.3), then

$$
(i \omega)^{3}+M_{1}(i \omega)^{2}+M_{2}(i \omega)+M_{3} e^{-i \omega \tau}+M_{2} M_{2}=0,
$$

separating the real and imaginary parts, we obtain

$$
\begin{gathered}
-M_{1} \omega^{2}+M_{3} \omega \sin (\omega \tau)+M_{1} M_{2}=0, \\
-\omega^{3}+M_{3} \omega \cos (\omega \tau)+M_{2} \omega=0 .
\end{gathered}
$$

It is easy to see from (2.6) and (2.7) that

$$
\omega^{6}+\left(M_{1}^{2}-2 M_{2}\right) \omega^{4}+\left(M_{2}^{2}-2 M_{1}^{2} M_{2}-M_{3}^{2}\right) \omega^{2}+M_{1}^{2} M_{2}^{2}=0 .
$$

Let $\omega^{2}=q$, then $(2.8)$ reduce to

$$
q^{3}+\left(M_{1}^{2}-2 M_{2}\right) q^{2}+\left(M_{2}^{2}-2 M_{1}^{2} M_{2}-M_{3}^{2}\right) q+M_{1}^{2} M_{2}^{2}=0 .
$$

Now, by Descartes' rule of sign, for an equation with real coefficients, non-existence of a positive root of (2.9) is that the coefficients are positive, namely, $M_{1}^{2}>2 M_{2}$ and $M_{2}^{2}>2 M_{1}^{2} M_{2}+M_{3}^{2}$. So $i \omega$ is not a root of (2.3). Then, the proof is complete.

Lemma 2.3 Assume that $\left(H_{1}\right),\left(H_{2}\right)$ and $\left(H_{3}\right)$ hold.

(1) If $\tau \in\left[0, \tau_{0}\right)$, then the positive equilibrium $E^{*}$ of system (1.2) is asymptotically stable;

(2) If $\tau>\tau_{0}$, then the positive equilibrium $E^{*}$ of system (1.2) is unstable;

(3) System (1.2) can undergo a Hopf bifurcation at the positive equilibrium $E^{*}$ when $\tau=\tau_{j}(j=0,1,2 \ldots)$, where $\tau_{j}$ is defined by $(2.5)$.

Proof Through (2.6) and (2.7), we can get (2.5) obtained. So, $j=0,(2.5)$ gives

$$
\tau_{0}=\frac{1}{\omega_{0}} \arccos \left[\frac{M_{1} \omega_{0}^{2}-M_{1} M_{2}}{\omega_{0}^{3}-M_{2} \omega_{0}}\right],
$$

which is the smallest time delay for which the stable solution becomes unstable. Thus the 
characteristic equation (2.3) has a pair of purely imaginary roots when $\omega=\omega_{0}$ and $\tau=\tau_{0}$.

Let $\lambda(\tau)=v(\tau)+i \omega(\tau)$ be the root of (2.3) such that when $\tau=\tau_{j}$ satisfying $v\left(\tau_{j}\right)=0$ and $\omega\left(\tau_{j}\right)=\omega_{0}$. Differentiating two sides of (2.3) with respect to $\tau$, we have

$$
\left[\operatorname{Re}\left(\frac{d \lambda}{d \tau}\right)^{-1}\right]_{\lambda=i \omega_{0}}=\frac{3 \omega_{0}^{4}+2\left(M_{2}^{2} M_{2}\right) \omega_{0}^{2}+M_{2}^{2}-2 M_{1}^{2} M_{2}-M_{3}^{2}}{M_{3}^{2} \omega_{0}^{2}} .
$$

In (2.9) denoting the left side by $\Omega$, and differentiation it at $q_{2}=\omega_{0}^{2}$. Combing with (2.10), we get

$$
\left[\operatorname{Re}\left(\frac{d \lambda}{d \tau}\right)^{-1}\right]_{\lambda=i \omega_{0}}=\left.\frac{1}{M_{3}^{2} \omega_{0}^{2}} \frac{d \Omega}{d q}\right|_{q=\omega_{0}^{2}}
$$

The sign of (2.11) is nonzero, so the characteristic (2.3) cannot have a multiple imaginary roots. Therefore, transversality condition holds. According to the bifurcation theorem for functional differential equation. One of the main results of this paper is as follows.

Theorem2.1 Assume that $\left(H_{1}\right),\left(H_{3}\right),\left(H_{3}\right)$ hold.

(a) Then the positive equilibrium $E^{*}$ of system (1.2) is asymptotically stable for all $\tau \geq 0$;

(b) If the above is hold, then the positive equilibrium $E^{*}$ of system (1.2) is asymptotically stable for $\tau \in\left[0, \tau_{0}\right)$;

(c) If $H_{1}$ and $H_{4}$ hold, then model system (2.1) is unstable for $\tau \geq \tau_{0}$. Hopf bifurcation occurs at $\tau=\tau_{0}$.

\section{Stability of bifurcated periodic solutions}

Now, we investigate the direction and stability of the periodic solutions bifurcated from the positive equilibrium $E^{*}$. Without loss of generality, we denote critical values $\tau=\tau_{j},(j=0,1,2 \ldots)$ by $\tau$ when the characteristic equation (2.3) has a pair of purely imaginary roots $\pm i \omega_{0}$, we denote $\tau$ as $\tau=\tilde{\tau}+\mu, \mu \in R$. Then $\mu=0$ is the Hopf bifurcation value of system (2.1).

Let $y_{1}(t)=u_{1}(t)-x_{1}^{*}, y_{2}(t)=u_{2}(t)-x_{2}^{*}, y_{3}(t)=u_{3}(t)-x_{3}^{*}$, then (2.1) reduces to

$$
\left\{\begin{array}{l}
y_{1}^{\prime}(t)=u_{1}^{\prime}(t)=-d y_{1}(t)-\beta y_{1}(t) y_{2}(t-\tau)-\beta y_{1}(t) x_{2}^{*} y_{2}(t-\tau), \\
y_{2}^{\prime}(t)=u_{2}^{\prime}(t)=\left(y_{2}(t)+x_{2}^{*}\right)\left(\beta y_{1}(t)-p y_{3}(t)\right), \\
y_{3}^{\prime}(t)=u_{3}^{\prime}(t)=c y_{2}(t)\left(x_{3}^{*}+y_{3}(t)\right) .
\end{array}\right.
$$

Let $W_{1}(t)=y_{1}(\tau t), W_{2}(t)=y_{2}(\tau t)$ and $W_{3}(t)=y_{3}(\tau t)$, then we have

$$
\left\{\begin{array}{l}
W_{1}^{\prime}(t)=y_{1}^{\prime}(t) \tau=\tau\left[-\left(d+\beta x_{2}^{*}\right) W_{1}(\tau t)-\beta W_{2}(t-1) x_{1}^{*}-\beta W_{1}(t) W_{2}(t-1)\right], \\
W_{2}^{\prime}(t)=y_{2}^{\prime}(t) \tau=\tau\left(W_{2}(t)+x_{2}^{*}\right)\left(\beta W_{1}(t)-p W_{3}(t)\right), \\
W_{3}^{\prime}(t)=y_{3}^{\prime}(t) \tau=\tau c W_{2}(t)\left(W_{3}(t)+x_{3}^{*}\right) .
\end{array}\right.
$$

Then, system (2.1) can be rewritten as the following functional differential in $C\left([1-, \infty), R^{3}\right)$ as

$$
W^{\prime}(t)=L_{\mu}(W(t))+F(\mu, W(t)),
$$

where $W(t)=\left(W_{1}(t), W_{2}(t), W_{3}(t)\right)^{T} \in R^{3}, L_{\mu}: C \rightarrow R$ and $F: R \times C \rightarrow R$ are defined respectively as follows.

$$
L_{\mu}(\phi)=(\tilde{\tau}+\mu)\left(\begin{array}{ccc}
-d-\beta x_{2}^{*} & 0 & 0 \\
\beta x_{2}^{*} & 0 & -p x_{2}^{*} \\
0 & c x_{3}^{*} & 0
\end{array}\right)\left(\begin{array}{l}
\phi_{1}(0) \\
\phi_{2}(0) \\
\phi_{3}(0)
\end{array}\right)+(\tilde{\tau}+\mu)\left(\begin{array}{ccc}
0 & -\beta x_{2}^{*} & 0 \\
0 & 0 & 0 \\
0 & 0 & 0
\end{array}\right)\left(\begin{array}{l}
\phi_{1}(-1) \\
\phi_{2}(-1) \\
\phi_{3}(-1)
\end{array}\right) \text {, }
$$

and

$$
F(\mu, W(t))=(\tilde{\tau}+\mu)\left(\begin{array}{c}
-\beta \phi_{1}(0) \phi_{2}(-1) \\
\beta \phi_{1}(0) \phi_{2}(0)-p \phi_{2}(0) \phi_{3}(0) \\
\left.c \phi_{2}(0) \phi_{3}(0)\right)
\end{array}\right),
$$

where $\phi(\theta)=\left(\phi_{1}(\theta), \phi_{2}(\theta), \phi_{3}(\theta)\right)^{T} \in C\left([-1,0], R^{3}\right)$. By Riesz representation theorem, there exist a 
matrix whose components are bounced variation function $\eta(\theta, \mu)$ for $\theta \in C\left([-1,0], R^{3}\right)$, such that

$$
L_{\mu} \phi=\int_{-1}^{0} \phi(\theta) \mathrm{d} \eta(\theta, \mu), \text { for } \theta \in C\left([-1,0], R^{3}\right) .
$$

For this representation, we can choose $\eta(\theta, \mu)$ as follows.

$$
\eta(\theta, \mu)=(\tilde{\tau}+\mu)\left(\begin{array}{ccc}
-d-\beta x_{2}^{*} & 0 & 0 \\
\beta x_{2}^{*} & 0 & -p x_{2}^{*} \\
0 & c x_{3}^{*} & 0
\end{array}\right) \delta(\theta)+(\tilde{\tau}+\mu)\left(\begin{array}{ccc}
0 & -\beta x_{2}^{*} & 0 \\
0 & 0 & 0 \\
0 & 0 & 0
\end{array}\right) \delta(\theta+1),
$$

where $\delta$ is the Dirac delta function i.e. $\delta(\theta)=\left\{\begin{array}{cc}0, & \theta \neq 0, \\ -1, & \theta=0 .\end{array}\right.$

For $\phi \in C\left([-1,0], R^{3}\right)$, we define

$$
A(\mu) \phi=\left\{\begin{array}{cc}
\frac{\mathrm{d} \phi}{\mathrm{d} \theta}, & -1 \leq \theta<0 \\
\int_{-1}^{0} \phi(\theta) \mathrm{d} \eta(\theta, \mu), & \theta=0
\end{array}\right.
$$

and

$$
R(\mu) \phi=\left\{\begin{array}{cc}
0, & -1 \leq \theta<0, \\
F(\theta, \mu), & \theta=0 .
\end{array}\right.
$$

From (3.8) and (3.9), the system (3.3) is equivalent to

$$
W_{t}^{\prime}=A(\mu) W_{t}+R(\mu) W_{t},
$$

where $W_{t}(\theta)=W(t+\theta)$, for $-1 \leq \theta<0$. For $\psi \in C\left([-1,0], R^{3}\right.$, the adjoint operator $A^{*}$ of $A$ is defined by

associate with a bilinear form

$$
A^{*}=\left\{\begin{array}{cc}
-\frac{\mathrm{d} \psi(s)}{d s}, & 0<s \leq 1, \\
\int_{-1}^{0} \psi(-s) \mathrm{d} \eta(s, 0), & s=0 .
\end{array}\right.
$$

$$
<\psi(s), \phi(s)>=\overline{\psi(0)} \phi(0)-\int_{\theta=-1}^{0} \int_{\xi=0}^{\theta}(\bar{\psi})^{T}(\xi-\theta) \mathrm{d} \eta(\theta) \phi(\xi) \mathrm{d} \xi,
$$

where $\eta(\theta)=\eta(\theta, 0)$. The normal form of eigenvector $l$ of $A$ belonging to the eigenvalue $i \omega_{0} \tilde{\tau}$ and the eigenvector $l^{*}$ of $A^{*}$ belonging to the eigenvalue $-i \omega_{0} \tilde{\tau}$. The eigenvector are given $A(0) l(\theta)=i \omega_{0} \tilde{\tau} l(\theta)$. Then,

$$
l(\theta)=\left(\alpha_{1}, \alpha_{2}, 1\right)^{T} e^{i \omega_{0} \tilde{\tau} \theta}, \quad \alpha_{1}=\frac{-\omega_{0}^{2}+c p x_{2}^{*} x_{3}^{*}}{c \beta x_{2}^{*} x_{3}^{*}}, \quad \alpha_{2}=\frac{i \omega_{0}}{c x_{3}^{*}}
$$

If $l(s)^{*}=D\left(\alpha_{1}^{*}, \alpha_{2}^{*}, 1\right)^{T} e^{i \omega_{0} \tilde{\tau} s}$, be the eigenvector of $A^{*}(0)$, similarly,

$$
A^{*}(0) l(s)^{*}=-i \omega_{0} \tilde{\tau} l^{*}(s), \quad \alpha_{1}^{*}=\frac{-\omega_{0}^{2}+c p x_{2}^{*} x_{3}^{*}}{p \beta x_{1}^{*} x_{2}^{*}} e^{i \omega_{0} \tilde{\tau}}, \quad \alpha_{2}^{*}=\frac{i \omega_{0}}{p x_{2}^{*}} .
$$

Since the property of adjoint, we have $\left\langle l^{*}(s), l(\theta)\right\rangle=1$, and $\left\langle l^{*}(s), \overline{l(\theta)}\right\rangle=0$, we get

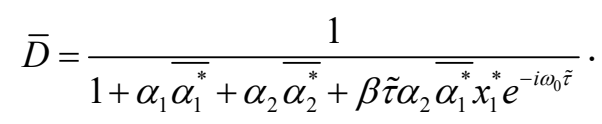

Let $N_{t}$ be the solution of (3.3), when $\mu=0$. Let's define

$$
z(t)=<l^{*}, N_{t}>; \quad G(t, \theta)=N_{t}(\theta)-2 \operatorname{Re}[z(t) l(\theta)] .
$$

On the center manifold $C_{0}$, we have

and

$$
G(t, \theta)=G(z(t), \overline{z(t)}, \theta)
$$

$$
G(z, \bar{z}, \theta)=G_{20}(\theta) \frac{z^{2}}{2}+G_{11}(\theta) z \bar{z}+G_{02}(\theta) \frac{\overline{z^{2}}}{2}+G_{30}(\theta) \frac{z^{3}}{6}+\ldots
$$


where $z$ and $\bar{z}$ are local coordinate for center manifold $C_{0}$, in the direction of $l^{*}$ and $l^{*}$. Note that $G$ is real if $N_{t}$ is real. We only study the real solutions. For solution $N_{t} \in C_{0}$ of (3.10), since $\mu=0$, then

$$
z^{\prime}(t)=<l^{*}, N_{t}^{\prime}>=i \omega_{0} \tilde{\tau} z+\overline{l^{*}} F(0, G(z, \bar{z}, 0))+2 \operatorname{Re}[z(t) l(\theta)] \triangleq i \omega_{0} \tilde{\tau} z+\overline{l^{*}} F_{0}(z, \bar{z}) .
$$

We rewrite the equation as

$$
z^{\prime}(t)=i \omega_{0} \tilde{\tau} z(t)+g(z, \bar{z})
$$

where

$$
g(z, \bar{z})=\overline{l^{*}} F_{0}(z, \bar{z})=g_{20} \frac{z^{2}}{2}+g_{11} z \bar{z}+g_{02} \frac{\overline{z^{2}}}{2}+g_{21} \frac{z^{2} \bar{z}}{2}+\ldots .
$$

It follows from (3.15) and (3.17) that

$$
\begin{aligned}
& N(t)=\left(N_{1 t}, N_{2 t}, N_{3 t}\right) \\
& =G_{20}(\theta) \frac{z^{2}}{2}+G_{11}(\theta) z \bar{z}+G_{02}(\theta) \frac{\overline{z^{2}}}{2}+\left(\alpha_{1}, \alpha_{2}, 1\right) e^{i \omega_{0} \tilde{\tau} \theta} z+\left(\tilde{\alpha}_{1}, \tilde{\alpha}_{2}, 1\right) e^{-i \omega_{0} \tilde{z} \theta} \tilde{z}+\ldots,
\end{aligned}
$$

and

$$
\begin{aligned}
& N_{1 t}(0)=G_{20}^{(1)}(0) \frac{z^{2}}{2}+G_{11}^{(1)}(0) z \bar{z}+G_{02}^{(1)}(0) \frac{\overline{z^{2}}}{\frac{2}{\bar{m}}}+\alpha_{1} z+\overline{\alpha_{1}} \bar{z}+\ldots \\
& N_{2 t}(0)=G_{20}^{(2)}(0) \frac{z^{2}}{2}+G_{11}^{(2)}(0) z \bar{z}+G_{02}^{(2)}(0) \frac{\overline{z^{2}}}{2}+\alpha_{2} z+\overline{\alpha_{2}} \bar{z}+\ldots \\
& N_{3 t}(0)=G_{20}^{(3)}(0) \frac{z^{2}}{2}+G_{11}^{(3)}(0) z \bar{z}+G_{02}^{(3)}(0) \frac{z^{2}}{2}+z+\bar{z}+\ldots \\
& N_{1 t}(-1)=G_{20}^{(1)}(-1) \frac{z^{2}}{2}+G_{11}^{(1)}(-1) z \bar{z}+G_{02}^{(1)}(-1) \frac{\overline{z^{2}}}{2}+\alpha_{1} z e^{-i \omega_{0} \tilde{\tau}}+\overline{\alpha_{1}} \bar{z} e^{i \omega_{0} \tilde{\tau}}+\ldots \\
& N_{2 t}(-1)=G_{20}^{(2)}(-1) \frac{z^{2}}{2}+G_{11}^{(2)}(-1) z \bar{z}+G_{02}^{(2)}(-1) \frac{\overline{z^{2}}}{\frac{2}{\bar{z}}}+\alpha_{2} z e^{-i \omega_{0} \tilde{\tau}}+\overline{\alpha_{2}} \bar{z} e^{i \omega_{0} \tilde{\tau}}+\ldots \\
& N_{3 t}(-1)=G_{20}^{(3)}(-1) \frac{z^{2}}{2}+G_{11}^{(3)}(-1) z \bar{z}+G_{02}^{(3)}(-1) \frac{z^{2}}{2}+z e^{-i \omega_{0} \tilde{\tau}}+\bar{z} e^{i \omega_{0} \tilde{\tau}}+\ldots
\end{aligned}
$$

then, we get

$$
\begin{gathered}
g(z, \bar{z})=\tilde{\tau} \bar{D}\left[-\alpha_{1}^{*} \beta\left(G_{20}^{(1)}(0) \frac{z^{2}}{2}+G_{11}^{(1)}(0) z \bar{z}+G_{02}^{(1)}(0) \frac{\overline{z^{2}}}{2}+\alpha_{1} z+\overline{\alpha_{1}} \bar{z}+\ldots\right)\right. \\
\left.\left(G_{20}^{(2)}(-1) \frac{z^{2}}{2}+G_{11}^{(2)}(-1) z \bar{z}+G_{02}^{(2)}(-1)\right) \frac{\overline{z^{2}}}{2}+\alpha_{2} z e^{-i \omega_{0} \tilde{\tau}}+\overline{\alpha_{2}} \bar{z} e^{i \omega_{0} \tilde{\tau}}+\ldots\right) \\
+\alpha_{2}^{*}\left[\beta\left(G_{20}^{(1)}(0) \frac{z^{2}}{2}+G_{11}^{(1)}(0) z \bar{z}+G_{02}^{(1)}(0) \frac{\overline{z^{2}}}{2}+\alpha_{1} z+\overline{\alpha_{1}} \bar{z}+\ldots\right)\right. \\
\left(G_{20}^{(2)}(0) \frac{z^{2}}{2}+G_{11}^{(2)}(0) z \bar{z}+G_{02}^{(2)}(0) \frac{\overline{z^{2}}}{2}+\alpha_{2} z+\overline{\alpha_{2}} \bar{z}+\ldots\right) \\
-p\left(G_{20}^{(2)}(0) \frac{z^{2}}{2}+G_{11}^{(2)}(0) z \bar{z}+G_{02}^{(2)}(0) \frac{\overline{z^{2}}}{2}+\alpha_{2} z+\overline{\alpha_{2}} \bar{z}+\ldots\right) \\
\left.\left(G_{20}^{(3)}(0) \frac{z^{2}}{2}+G_{11}^{(3)}(0) z \bar{z}+G_{02}^{(3)}(0) \frac{\overline{z^{2}}}{2}+z+\bar{z}+\ldots\right)\right] \\
+c\left(G_{20}^{(2)}(0) \frac{z^{2}}{2}+G_{11}^{(2)}(0) z \bar{z}+G_{02}^{(2)}(0) \frac{z^{2}}{2}+\alpha_{2} z+\overline{\alpha_{2}} \bar{z}+\ldots\right) \\
\left.\left(G_{20}^{(3)}(0) \frac{z^{2}}{2}+G_{11}^{(3)}(0) z \bar{z}+G_{02}^{(3)}(0) \frac{\overline{z^{2}}}{2}+z+\bar{z}+\ldots\right)\right] .
\end{gathered}
$$

Comparing the coefficients with (3.19), we have 


$$
\begin{aligned}
& g_{20}=2 \tilde{\tau} \bar{D}\left(-\beta \alpha_{1}^{*} \alpha_{2} \overline{\alpha_{1}^{*}} e^{-i \omega_{0} \tilde{\tau}}+\beta \alpha_{1} \alpha_{2} \alpha_{2}^{*}-p \alpha_{2} \alpha_{2}^{*}+c \alpha_{2}\right) \\
& g_{11}=2 \tilde{\tau} \bar{D}\left[\left(-\beta \alpha_{1}^{*}\right)\left(\alpha_{1} \overline{\alpha_{2}} e^{i \omega_{0} \tilde{\tau}}+\overline{\alpha_{1}} \alpha_{2} e^{-i \omega_{0} \tilde{\tau}}\right)+\overline{\alpha_{2}^{*}}\left(\beta\left(\alpha_{1} \overline{\alpha_{2}}+\beta \alpha_{2} \overline{\alpha_{1}}\right)\right.\right. \\
& -p\left(\beta\left(\alpha_{2}+\overline{\alpha_{2}}\right)\right)+2 c\left(\beta\left(\alpha_{2}+\overline{\alpha_{2}}\right)\right] \\
& g_{02}=2 \tilde{\tau} \bar{D}\left[-\beta \overline{\alpha_{1}} \overline{\alpha_{2}} \overline{\alpha_{1}^{*}} e^{i \omega_{0} \tilde{\tau}}+\overline{\alpha_{2}^{*}}\left(\beta \overline{\alpha_{1}} \overline{\alpha_{2}}-p \overline{\alpha_{2}}\right)+c \overline{\alpha_{2}}\right] \\
& g_{21}=2 \tilde{\tau} \bar{D}\left(\left(-\beta \overline{\alpha_{1}^{*}}\left(\frac{1}{2} G_{20}^{(1)}(0) \overline{\alpha_{2}} e^{i \omega_{0} \tilde{\tau}}+G_{11}^{(1)}(0) \alpha_{2} e^{-i \omega_{0} \tilde{\tau}}+\alpha_{1} G_{11}^{(2)}(-1)+\frac{1}{2} \overline{\alpha_{1}} G_{20}^{(2)}(-1)\right)\right.\right. \\
& \left.+\overline{\alpha_{2}^{*}}\left[\beta\left(\frac{1}{2}\right) G_{20}^{(1)}(0) \overline{\alpha_{1}}+G_{11}^{(1)}(0) \alpha_{2}+G_{11}^{(2)}(0) \alpha_{1}+\frac{1}{2}\right) G_{20}^{(2)}(0) \overline{\alpha_{1}}\right) \\
& \left.\left.\left.-p\left(\frac{1}{2}\right) G_{20}^{(2)}(0)+G_{11}^{(2)}(0)+G_{11}^{(3)}(0) \alpha_{2}+\frac{1}{2}\right) G_{20}^{(3)}(0) \overline{\alpha_{2}}\right)\right] \\
& \left.\left.\left.+c\left(\frac{1}{2}\right) G_{20}^{(2)}(0)+G_{11}^{(2)}(0)+G_{11}^{(3)}(0) \alpha_{2}+\frac{1}{2}\right) G_{20}^{(3)}(0) \overline{\alpha_{2}}\right)\right) .
\end{aligned}
$$

In order to determinate $g_{21}$, we focus on the computation of $G_{20}(\theta)$ and $G_{11}(\theta)$. From (3.10), (3.15) and (3.19), we can obtain

$$
G^{\prime}=N_{t}-z^{\prime} l-\bar{z}^{\prime} \bar{l}=\left\{\begin{array}{cc}
A(0) G(t, \theta)-2 \operatorname{Re}\left[\overline{l^{*}(0)} F_{0} l(\theta)\right], & -1 \leq \theta<0, \\
A(0) G(t, \theta)-2 \operatorname{Re}\left[\overline{l^{*}(0)} F_{0} l(\theta)\right]+F_{0}, & \theta=0 .
\end{array}\right.
$$

Let

where

$$
G^{\prime}=A(0) G(t, \theta)+Q(z, \bar{z}, \theta),
$$

Differentiating two sides of (3.17) with respect to $t$, we have

$$
G^{\prime}=G_{z} z^{\prime}+G_{\bar{z}} \bar{z}^{\prime}
$$

According to (3.25) and (3.26), we obtain

$$
\left(A(0)-2 i \omega_{0} \tilde{\tau} I\right)=-Q_{20}(\theta), \quad A(0) N(\theta)=-Q_{11}(\theta)
$$

From (3.24), when $-1 \leq \theta<0$, we get

$$
Q(z, \bar{z}, \theta)=-\bar{l}^{*}(0) F_{0} l(\theta)-{\overline{F_{0}} l(0)}^{*} \overline{l(\theta)}=-g(z, \bar{z}) l(\theta)-\overline{g(z, \bar{z})} \overline{l(\theta)} .
$$

Thus,

We can obtain that

$$
Q_{20}(\theta)=-g_{20} l(\theta)-\overline{g_{02}} \overline{l(\theta)}, Q_{11}(\theta)=-g_{11} l(\theta)-\overline{g_{11} l(\theta)} .
$$

then

$$
G_{20}^{\prime}=2 i \omega_{0} \tilde{\tau} G_{20}+g_{20} l(\theta)+\overline{g_{20}} \overline{l(\theta)},
$$

Similarly,

$$
G_{20}(\theta)=\frac{i g_{20} l(0)}{\omega_{0} \tilde{\tau}} e^{i \omega_{0} \tilde{\tau} \theta}+\frac{\overline{i \overline{g_{02}} l(0)}}{3 \omega_{0} \tilde{\tau}} e^{-i \omega_{0} \tilde{\tau} \theta}+E_{1} e^{2 i \omega_{0} \tilde{\tau} \theta}
$$

$$
G_{11}(\theta)=-\frac{i g_{11} l(0)}{\omega_{0} \tilde{\tau}} e^{i \omega_{0} \tilde{\tau} \theta}+\frac{\overline{i g_{02}} \overline{l(0)}}{3 \omega_{0} \tilde{\tau}} e^{-i \omega_{0} \tilde{\tau} \theta}+E_{2} e^{2 i \omega_{0} \tilde{\tau} \theta},
$$

Next, we focus on the computation of $E_{1}$ and $E_{2}$. From (3.27), we achieve

$$
\int_{-1}^{0} \mathrm{~d} \eta(\theta) G_{20}(\theta)=2 i \omega_{0} \tilde{\tau} G_{20}(\theta)-Q_{20}(\theta), \int_{-1}^{0} \mathrm{~d} \eta(\theta) G_{11}(\theta)=-Q_{11}(\theta) .
$$

where $\eta(\theta)=\eta(0, \theta)$. By (3.22), we have

$$
Q_{20}(0)=g_{20} l(0)-\overline{g_{02}} l(0)+2 \tilde{\tau}\left(\begin{array}{c}
-\beta \alpha_{1} \alpha_{2} e^{-i \omega_{0} \tilde{\tau}} \\
\beta \alpha_{1} \alpha_{2}-p \alpha_{2} \\
c \alpha_{2}
\end{array}\right),
$$

and 


$$
Q_{11}(0)=-g_{11} l(0)-\overline{g_{11}} \overline{l(0)}+2 \tilde{\tau}\left(\begin{array}{c}
-\beta\left(\alpha_{1} \overline{\alpha_{2}} e^{i \omega_{0} \tilde{\tau}}+\overline{\alpha_{1}} \alpha_{2} e^{-i \omega_{0} \tilde{\tau}}\right) \\
\beta\left(\alpha_{1} \overline{\alpha_{2}}+\overline{\alpha_{1}} \alpha_{2}\right)-p\left(\alpha_{2} \overline{\alpha_{2}}\right) \\
c\left(\alpha_{2} \overline{\alpha_{2}}\right)
\end{array}\right) .
$$

Then

We get

$$
\left[i \omega_{0} \tilde{\tau} I-i n t_{-1}^{0} e^{i \omega_{0} \tilde{\theta} \theta} \mathrm{d} \eta(\theta)\right] l(\theta)=0,\left[-i \omega_{0} \tilde{\tau} I-i n t_{-1}^{0} d e^{-i \omega_{0} \tilde{\theta} \theta} \eta(\theta)\right] l(\theta)=0
$$

It follows that

$$
\left(2 i \omega_{0} \tilde{\tau} I-i n t_{-1}^{0} e^{2 i \omega_{0} \tilde{\theta} \theta} \mathrm{d} \eta(\theta)\right) l(\theta)=2 \tilde{\tau}\left(\begin{array}{c}
-\beta \alpha_{1} \alpha_{2} e^{-i \omega_{0} \tilde{\tau}} \\
\beta \alpha_{1} \alpha_{2}-p \alpha_{2} \\
c \alpha_{2}
\end{array}\right) .
$$

$$
\begin{gathered}
E_{1}^{(1)}=\frac{2}{B}\left|\begin{array}{ccc}
-\beta \alpha_{1} \alpha_{2} e^{-i \omega_{0} \tau} & \beta x_{1}^{*} e^{-2 i \omega_{0} \tilde{\tau}} & 0 \\
\beta \alpha_{1} \alpha_{2}-p \alpha_{2} & 2 i \omega_{0} & p x_{2}^{*} \\
c \alpha_{2} & -c x_{3}^{*} & 2 i \omega_{0}
\end{array}\right|, E_{1}^{(2)}=\frac{2}{B}\left|\begin{array}{ccc}
2 i \omega_{0}+d+\beta x_{2}^{*} & -\beta \alpha_{1} \alpha_{2} e^{-i \omega_{0} \tilde{\tau}} & 0 \\
-\beta \alpha_{2}^{*} & \beta \alpha_{1} \alpha_{2}-p \alpha_{2} & p x_{2}^{*} \\
0 & c \alpha_{2} & 2 i \omega_{0}
\end{array}\right|, \\
E_{1}^{(3)}=\frac{2}{B}\left|\begin{array}{ccc}
2 i \omega_{0}+d+\beta x_{2}^{*} & \beta x_{1}^{*} e^{-2 i \omega_{0} \tilde{\tau}} & -\beta \alpha_{1} \alpha_{2} e^{-i \omega_{0} \tilde{\tau}} \\
-\beta x_{2}^{*} & 2 i \omega_{0} & \beta \alpha_{1} \alpha_{2}-p \alpha_{2} \\
0 & -c x_{3}^{*} & c \alpha_{2}
\end{array}\right|, B=\left|\begin{array}{ccc}
2 i \omega_{0}+d+\beta x_{2}^{*} & \beta x_{1}^{*} e^{-2 i \omega_{0} \tilde{\tau}} & 0 \\
-\beta x_{2}^{*} & 2 i \omega_{0} & p x_{2}^{*} \\
0 & -c x_{3}^{*} & 2 i \omega_{0}
\end{array}\right|
\end{gathered}
$$

Similarly,

$$
\begin{gathered}
E_{2}^{(1)}=\frac{2}{B^{\prime}}\left|\begin{array}{ccc}
-\beta\left(\alpha_{1} \overline{\alpha_{2}} e^{i \omega_{0} \tilde{\tau}}+\alpha_{2} \overline{\alpha_{1}} e^{i \omega_{0} \tilde{\tau}}\right) & \beta x_{1}^{*} & 0 \\
\left.c \alpha_{2}+\overline{\alpha_{2}}\right)-p\left(\alpha_{2}+\overline{\alpha_{2}}\right) & 0 & p x_{2}^{*} \\
E_{2}^{(2)}=\frac{2}{B^{\prime}}\left|\begin{array}{ccc}
d+\beta x_{2}^{*} & -\beta\left(\alpha_{1} \overline{\alpha_{2}} e^{i \omega_{0} \tilde{\tau}}+\alpha_{2} \overline{\alpha_{1}} e^{i \omega_{0} \tilde{\tau}}\right) & 0
\end{array}\right|, \\
-\beta x_{2}^{*} & \beta\left(\alpha_{1} \overline{\alpha_{2}}+\alpha_{2} \overline{\alpha_{1}}\right)-p\left(\alpha_{2}+\overline{\alpha_{2}}\right) & \beta\left(\alpha_{1} \overline{\alpha_{2}}+\alpha_{2} \overline{\alpha_{1}}\right)-p\left(\alpha_{2}+\overline{\alpha_{2}}\right) \\
0 & c \alpha_{2}+\overline{\alpha_{2}} & 0
\end{array}\right|, \\
E_{2}^{(3)}=\frac{2}{B^{\prime}}\left|\begin{array}{ccc}
d+\beta x_{2}^{*} & \beta x_{1}^{*} & -\beta\left(\alpha_{1} \overline{\alpha_{2}} e^{i \omega_{0} \tilde{\tau}}+\alpha_{2} \overline{\alpha_{1}} e^{i \omega_{0} \tilde{\tau}}\right) \\
-\beta x_{2}^{*} & 0 & \beta \alpha_{1} \alpha_{2}-p \alpha_{2} \\
0 & -c x_{3}^{*} & c \alpha_{2}+\overline{\alpha_{2}}
\end{array}\right|, B^{\prime}=\left|\begin{array}{ccc}
d+\beta x_{2}^{*} & \beta x_{1}^{*} & 0 \\
-\beta x_{2}^{*} & 0 & p x_{2}^{*} \\
0 & -c x_{3}^{*} & 0
\end{array}\right| .
\end{gathered}
$$

Thus, we can calculate the following values

$$
\begin{aligned}
C_{1}(0) & =\frac{i}{2 \omega_{0} \tilde{\tau}}\left(g_{20} g_{11}-2\left|g_{11}\right|^{2}-\frac{\left|g_{02}\right|^{2}}{3}\right)+\frac{g_{21}}{2}, \\
\mu_{2} & =-\frac{\operatorname{Re} C_{1}(0)}{\operatorname{Re} \lambda^{\prime}(\tilde{\tau})}, \beta_{2}=\operatorname{Re} C_{1}(0), \quad T_{2}=-\frac{\operatorname{Im} C_{1}(0)+\mu_{2} \operatorname{Im} \lambda^{\prime}(\tilde{\tau})}{\omega_{0} \tilde{\tau}},
\end{aligned}
$$

which determine the qualities of furcated periodic solutions in the center manifold at the critical value $\tilde{\tau}$. According to the center manifold theorem, we have the following theorem which is the main result of this paper.

Theorem 3.1 Assume that $\left(H_{1}\right),\left(H_{2}\right),\left(H_{3}\right)$ and $\left(H_{4}\right)$ hold.

$\left(Y_{1}\right) \mu_{2}$ determine the direction of Hopf bifurcation. If $\mu_{2}>0\left(\mu_{2}<0\right)$, then the Hopf bifurcation is supercritical (subcritical);

$\left(Y_{2}\right) \beta_{2}$ determine the stability of bifurcated solutions. If $\beta_{2}>0\left(\beta_{2}<0\right)$, then the bifurcated solutions are stable (unstable);

$\left(Y_{3}\right) T_{2}$ determine the periodic of bifurcated solutions. If $T_{2}>0\left(T_{2}<0\right)$, then the periodic increases (decreases).

\section{References}

[1] Hu, Q., Hu, Z., Liao F. (2016) Stability and Hopf bifurcation in a HIV-1 infection model with delays and logistic growth. Mathematics and Computers in Simulation, 128, 26-41. 
[2] Xiao, M., Zheng, W.X., Jiang, G., et al. (2015) Undamped oscillations generated by Hopf bifurcations in fractional-order recurrent neural networks with Caputo derivative, IEEE transactions on neural networks and learning systems, 26(12), 3201-3214.

[3]Deshpande, A.S., Daftardar, G.V., Sukale, Y.V. (2017) On Hopf bifurcation in fractional dynamical systems. Chaos, Solitons \& Fractals, 98, 189-198.

[4] Gopalsamy, K., Zhang, B.G. (1988) On a neutral delay logistic equation, Dynam. Stability Systems, 2, 183-195.

[5]Wang, T., Hu, Z., Liao, F. (2014) Stability and Hopf bifurcation for a virus infection model with delayed humoral immunity response. Journal of Mathematical Analysis and Applications, 411(1), 63-74.

[6]Song, Y., Zhang, T., Peng, Y. (2016) Turing-Hopf bifurcation in the reaction-diffusion equations and its applications. Communications in Nonlinear Science and Numerical Simulation, 33, 229-258.

[7] Huang C, Cao J, Xiao M, et al. (2018) Effects of time delays on stability and Hopf bifurcation in a fractional ring-structured network with arbitrary neurons, Communications in Nonlinear Science and Numerical Simulation, 57, 1-13. 